\title{
Deaths Associated with Pneumonic Plague, 1946-2017
}

\author{
Alex P. Salam, ${ }^{1}$ Amanda Rojek, ${ }^{1}$ Erhui Cai, Mihaja Raberahona, Peter Horby
}

The death rate for persons with treated pneumonic plague is often reported as $50 \%$, but firm evidence for this figure is minimal. We conducted a meta-analysis of articles reporting the death rate for persons treated for pneumonic plague. The rate was $17 \%$, substantially lower than the frequently cited $50 \%$.

Versinia pestis, the causative agent of plague, is a 1 Tier 1 select agent because of the high case-fatality rate associated with pneumonic plague and its potential as a bioterrorism agent in aerosolized form (https: / / emergency.cdc.gov/agent/agentlistcategory.asp). The death rate for persons with untreated primary pneumonic plague was reported to be almost $100 \%$ (1); the death rate for persons treated for primary pneumonic plague was $50 \%$ (1). Overall, the death rate for persons treated for primary pneumonic plague was high despite the sensitivity of $Y$. pestis to aminoglycosides, quinolones, and tetracyclines $(2,3)$ and the relatively good penetration of some of these antimicrobial drugs into lungs (4,5). During the 2017 Madagascar pneumonic plague outbreak, the observed death rate for treated persons appeared to be substantially lower than that reported in the literature (6). Many articles that quoted a $50 \%$ death rate for treated primary pneumonic plague were cited in a 2000 study by Ratsitorahina et al. (7), which described a small outbreak in Madagascar in 1997. The article indicated that the data showed an overall death rate of $53 \%$ but did not state the number of deaths. However, the death rate for treated persons with confirmed or probable plague was $10 \%$. On reviewing

Author affiliations: United Kingdom Public Health Rapid Support

Team, London, UK (A.P. Salam); Centre for Tropical Medicine and Global Health, University of Oxford, Oxford, UK (A.P. Salam,

E. Cai, M. Raberahona, P. Horby); Centre Hospitalier

Befelatanana, Antananarivo, Madagascar (M. Raberahona);

Centre for Integrated Critical Care, University of Melbourne,

Melbourne, Victoria, Australia (M. Raberahona) reports that cited Ratsitorahina et al., we identified 9 studies that referenced $50 \%$ of persons treated for pneumonic plague who died, 1 study that referenced $40 \%$, and none referencing lower rates. One was a review cited 9 times about persons treated for primary pneumonic plague for whom the death rate was $50 \%$. We identified 6 reports that stated but did not reference a $50 \%$ death rate for persons treated for pneumonic plague.

\section{The Study}

To address the lack of evidence supporting the frequently cited $50 \%$ death rate for persons treated for primary pneumonic plague, we conducted a systematic review and meta-analysis. We followed PRISMA (Preferred Reporting Items for Systematic Reviews and Meta-Analyses, http://www.prisma-statement.org) and MOOSE (Meta-analysis of Observational Studies in Epidemiology [8]) guidelines. The study was prospectively registered on PROSPERO (CRD42018086223) (https://www.crd.york.ac.uk/PROSPERO).

We searched PubMed and Embase covering 1946-2017 using the search terms "Yersinia pestis" or "plague" and "pneumon*" and limited our search to human data. We searched references and included articles describing death (within a 28-day period from illness onset) among patients with confirmed, probable, and suspected primary or undifferentiated (i.e., primary or secondary not distinguished pneumonic plague, 1999 World Health Organization case definition, https://www.who.int/csr/resources/publications/ plague/WHO_CDS_CSR_EDC_99_2_EN/en/). We did not restrict by study type, language, or minimum patient number.

Two authors reviewed and extracted data; a third author resolved any disagreements. Data fields extracted included year and country of the outbreak, number of patients who survived and died (stratified by antimicrobial drug status), number of patients receiving different antimicrobial

\footnotetext{
${ }^{1}$ These co-first authors contributed equally to this article.
} 
drug classes, time to antimicrobial drug administration, and receipt of plague vaccination or prophylaxis (these patients were excluded). We calculated the risk from the number of events and participants in each group.

We performed a meta-analysis using a binomialspecific method. We assessed heterogeneity using the $\chi^{2}$ test and quantified results with the $I^{2}$ statistic. In addition, we preplanned 2 sensitivity analyses to examine whether our estimation of death was influenced by the inclusion of specific articles (pneumonic plague was not confirmed as primary disease or patients with suspected and probable disease). We conducted statistical analysis using $R$ version 3.6.0 ( $R$ Project, https:/ / www.r-project.org).

We reviewed 362 articles (Appendix Figure 1, https:// wwwnc.cdc.gov/EID/article/26/10/191270-App1.pdf). We described 1,107 patients in 44 articles (Appendix Table). Twenty-nine articles reported antimicrobial drug use in 108 patients with confirmed or probable pneumonic plague. For pneumonic plague patients receiving antimicrobial drug therapy, the pooled death rate was 17\% (95\% CI 8\%-31\%; $I^{2}=47 \%$ ) (Appendix Figure 2). Pneumonic plague patients who did not receive antimicrobial drug therapy had a pooled death rate of 98\% (95\% CI 73\%-100\%; $\mathrm{I}^{2}=47 \%$ ) (Appendix Figure 3). Pneumonic plague patients for whom antimicrobial drug status was unknown had a pooled death rate of $46 \%$ (95\% CI 32\%-61\%) (Appendix Figure 4). Heterogeneity was significant $\left(I^{2}=91 \%\right.$; $\mathrm{p}<0.01)$. The pooled death rates were similar when sensitivity analysis was conducted (Table). Antimicrobial drugs in the reports were aminoglycosides (90 courses), quinolones (24 courses), sulfonamides (22 courses), chloramphenicol (16 courses), tetracyclines (14 courses), and cotrimoxazole (3 courses). Six reports described time to from admission to antimicrobial drug administration, but the nonstandardized reporting precluded stratification by this measure.

\begin{tabular}{|c|c|c|}
\hline Antimicrobial drug use & $\begin{array}{c}\text { Confirmed cases, \% } \\
(95 \% \mathrm{Cl})\end{array}$ & $\begin{array}{c}\text { Total cases, \% } \\
(95 \% \mathrm{Cl})\end{array}$ \\
\hline \multicolumn{3}{|l|}{ Treated } \\
\hline Primary plague & $27(14-47)$ & $6(1-31)$ \\
\hline Undifferentiated & $28(6-72)$ & $6(1-31)$ \\
\hline \multicolumn{3}{|l|}{ Not treated } \\
\hline Primary plague & $94(82-98)$ & $99(22-100)$ \\
\hline Undifferentiated & No data & $100^{*}$ \\
\hline \multicolumn{3}{|l|}{ Unknown } \\
\hline Primary plague & No data & $29(13-51)$ \\
\hline Undifferentiated & $42(23-64)$ & $51(31-71)$ \\
\hline
\end{tabular}

\section{Conclusions}

Our meta-analysis identified a $17 \%$ death rate for persons treated for pneumonic plague, in contrast to the $50 \%$ often reported in the literature. The death rate for the 2017 Madagascar outbreak was published after we completed our systematic review but is consistent with our findings (25\% in confirmed cases) (9). These figures compare with $13.6 \%$ for patients who died in the hospital of community-acquired pneumonia; $12.3 \%$ who died of Streptococcus pneumoniae infection; $14.7 \%$ who died of Legionella species infection; and $32 \%-61 \%$ who died of Staphylococcus aureus, Escherichia coli, Klebsiella species, or Pseudomonas aeruginosa infections (10). However, persons who died of other etiologic causes were predominantly elderly and had underlying conditions (10).

Our review indicated insufficient standardized data to stratify death by time from symptom onset to antimicrobial drug administration. The literature we assessed often stated that pneumonic plague is fatal in almost all patients who start antimicrobial drugs $>24$ hours after symptom onset. Generally, descriptions cite either 1 article, in which 11 patients treated within 24 hours survived and 2 treated after 24 hours died (11), or a handful of isolated case reports. However, case reports and series also exist in which patients survived despite starting antimicrobial drugs $>24$ hours after symptom onset (12-14).

An accurate estimate of death is crucial for several reasons. First, it is helpful for public health planning during outbreaks, including the allocation of healthcare resources and the development of social mobilization campaigns. The commonly reported high death rate associated with primary pneumonic plague contributes to fear and panic among healthcare workers and the public. For example, anecdotal reports indicating concerns during the Madagascar outbreak were the following: healthcare workers taking continuous antimicrobial drug prophylaxis, mass public use of over-the-counter antimicrobial drugs, asymptomatic persons visiting the hospital, and sick persons avoiding the hospital. Accurate assessment of death is also essential for clinical trial design. For example, the required sample size would be 134 (power $0.8, a=0.025$ ) for a binary outcome superiority trial in which the death rate in the control arm was $50 \%$ and the intervention was assumed to reduce death by $50 \%$ (similar to the assumptions in a clinical trial of gentamicin vs. doxycycline in Tanzania in 2002) (15). However, a sample size of 476 would be required in a trial in which the death rate in the control arm was $20 \%$. A sample size renders a superiority trial unfeasible. Even during the Madagascar outbreak, the largest 
outbreak of pneumonic plague this century, the final number of confirmed pneumonic plague patients was only $32(9)$.

The major limitation of our meta-analysis is the sporadic reporting of clinical data and the relatively small number of cases for which antimicrobial drugs treatment status was described. Reporting bias in the literature also is likely, and pneumonic plague patients who survive are more likely than those who do not to be reported. Nonetheless, data indicate that the percentage of persons who die of treated pneumonic plague appears to be substantially lower than is frequently reported in the literature.

\section{Acknowledgments}

We thank Freya Shearer for assisting with statistical programming.

The UK Public Health Rapid Support Team is funded by UK aid from the Department of Health and Social Care and is jointly run by Public Health England and the London School of Hygiene \& Tropical Medicine. The University of Oxford and King's College London are academic partners. P.H. is supported by funding from the Department for International Development and the Welcome Trust (215091/Z/18/Z) and the Bill \& Melinda Gates Foundation (OPP1209135).

\section{About the Author}

Dr. Salam is the clinical researcher for the United Kingdom Public Health Rapid Support Team. His primary research interests include clinical research in epidemic prone diseases. Dr. Rojek is senior clinical fellow at the Centre for Integrated Critical Care, University of Melbourne. Her primary research interests include clinical research in epidemic-prone diseases.

\section{References}

1. Prentice MB, Rahalison L. Plague. Lancet. 2007;369:1196-207. https:// doi.org/10.1016/S0140-6736(07)60566-2

2. Centers for Disease Control and Prevention. Recommended antibiotic treatment for plague [cited 2015 Aug 25]. https:/ / www.cdc.gov/ plague/resources/Recommendedantibiotics-for-plague-web-site-rev-Jan2018-P.pdf

3. Wendte JM, Ponnusamy D, Reiber D, Blair JL, Clinkenbeard KD.In vitro efficacy of antibiotics commonly used to treat human plague against intracellular Yersinia pestis. Antimicrob Agents Chemother. 2011;55:3752-7. https:/ / doi.org/10.1128/AAC.01481-10
4. Honeybourne D. Antibiotic penetration into lung tissues. Thorax. 1994;49:104-6. https:/ / doi.org/10.1136/thx.49.2.104

5. Valcke $Y$, Pauwels R, Van der Straeten M. Pharmacokinetics of antibiotics in the lungs. Eur Respir J. 1990;3:715-22.

6. Salam AP, Raberahona M, Andriantsalama P, Read L, Andrianarintsiferantsoa F, Razafinambinintsoa T, et al. Factors influencing atypical clinical case presentations during the 2017 Madagascar pneumonic plague outbreak: a prospective cohort study. Am J Trop Med Hyg. 2020;102:1309-15. https://doi.org/10.4269/ajtmh.19-0576

7. Ratsitorahina M, Chanteau S, Rahalison L, Ratsifasoamanana L, Boisier P. Epidemiological and diagnostic aspects of the outbreak of pneumonic plague in Madagascar. Lancet. 2000;355:111-3. https:/ / doi.org/ 10.1016/S0140-6736(99)05163-6

8. Stroup DF, Berlin JA, Morton SC, Olkin I, Williamson GD, Rennie D, et al. Meta-analysis of observational studies in epidemiology: a proposal for reporting. Meta-analysis Of Observational Studies in Epidemiology (MOOSE) group. JAMA. 2000;283:2008-12. https://doi.org/10.1001/ jama.283.15.2008

9. Randremanana R, Andrianaivoarimanana V, Nikolay B, Ramasindrazana B, Paireau J, Ten Bosch QA, et al. Epidemiological characteristics of an urban plague epidemic in Madagascar, August-November, 2017: an outbreak report. Lancet Infect Dis. 2019;19:537-45. https:/ / doi.org/10.1016/ S1473-3099(18)30730-8

10. Fine MJ, Smith MA, Carson CA, Mutha SS, Sankey SS, Weissfeld LA, et al. Prognosis and outcomes of patients with community-acquired pneumonia. A meta-analysis. JAMA. 1996;275:134-41. https://doi.org/10.1001/ jama.1996.03530260048030

11. McCrumb FR Jr, Mercier S, Robic J, Bouillat M, Smadel JE, Woodward TE, et al. Chloramphenicol and terramycin in the treatment of pneumonic plague. Am J Med. 1953;14:284-93. https://doi.org/10.1016/0002-9343(53)90040-0

12. Begier EM, Asiki G, Anywaine Z, Yockey B, Schriefer ME, Aleti $\mathrm{P}$, et al. Pneumonic plague cluster, Uganda, 2004. Emerg Infect Dis. 2006;12:460-7. https:/ / doi.org/10.3201/ eid1203.051051

13. Donaires LF, Céspedes M, Valencia P, Salas JC, Luna ME, Castañeda A, et al. [Primary pneumonic plague with nosocomial transmission in La Libertad, Peru 2010] [in Spanish]. Rev Peru Med Exp Salud Publica. 2010;27:326-36. https://doi.org/10.1590/S1726-46342010000300004

14. Luo H, Dong X, Li F, Xie X, Song Z, Shao Z, et al. A cluster of primary pneumonic plague transmitted in a truck cab in a new enzootic focus in China. Am J Trop Med Hyg. 2013;88:923-8. https://doi.org/10.4269/ ajtmh.12-0163

15. Mwengee W, Butler T, Mgema S, Mhina G, Almasi Y, Bradley C, et al. Treatment of plague with gentamicin or doxycycline in a randomized clinical trial in Tanzania. Clin Infect Dis. 2006;42:614-21. https:/ / doi.org/10.1086/500137

Address for correspondence: Peter Horby, University of Oxford, Center for Tropical Medicine and Global Health, Roosevelt Dr., Oxford, OX3 7FZ, UK; email: peter.horby@ndm.ox.ac.uk 